DOI: $10.3901 / J M E .2019 .09 .183$

\title{
基于偏心式变曲率沟槽的高精度球体加工 理论与试验研究 ${ }^{*}$
}

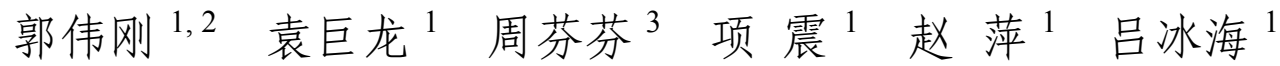

(1. 浙江工业大学超精密加工研究中心 杭州 310014;

2. 杭州职业技术学院 杭州 310018;

3. 台州学院机械工程学院 台州 318000)

\begin{abstract}
摘要: 提出基于偏心式变曲率沟槽的高精度球体批量加工方法, 加工过程中球体运动状态随磨盘沟槽曲率半径的变化而变化, 送料机构将球体从磨盘外沿的出料口依序送至磨盘中心的入料口进行循环加工。基于纯滚动运动假设, 建立单颗球体几何运动 学模型, 通过旋转坐标变换计算并仿真球面加工轨迹。在自主研发的偏心式变曲率沟槽加工系统上进行加工试验, 对球体运动 的宏观验证试验结果与仿真结果一致, 证明了所建的运动学模型的有效性。在加工试验研磨阶段球体的批直径变动量、球度偏 差及表面粗粘度偏差均得到明显改善并逐渐收玫, 抛光阶段后得到单颗球体最好的球度值为 $0.114 \mu \mathrm{m}$, 表面粗粘度 $R a$ 为 $9 \mathrm{~nm}$ 。 关键词: 运动学; 加工轨迹; 一致性; 偏心式变曲率沟槽; 精密球
\end{abstract}

中图分类号: TH145; TH140

\section{Theoretical and Experimental Research on Processing Balls with Eccentric Variable-radius V-groove}

\author{
GUO Weigang $^{1,2}$ YUAN Julong ${ }^{1}$ ZHOU Fenfen ${ }^{3} \quad$ XIANG Zhen $^{1}$ ZHAO Ping ${ }^{1}$ LÜ Binghai ${ }^{1}$ \\ (1. Ultra-precision Machining Center, Zhejiang University of Technology, Hangzhou 310014; \\ 2. Hangzhou Vocational and Technical College, Hangzhou 310018;
} 3. School of Mechanical Engineering, Taizhou University, Taizhou 318000)

\begin{abstract}
A new batch processing method for precision balls with eccentric variable-radius groove plate (EVGP) is proposed. In this method the motion state of ball is continuously changed with the variation of the groove radius as the ball moving along the groove. Balls can be one by one from the outlet along processing plate edge to the inlet in the center of plate by employed cycling feeding transporter. Based on the assumption of pure rolling motion, the geometric kinematic model of a single ball is built, and then the processing trajectory on the spherical surface can be obtained by rotation coordinate transformation. Processing experiment is carried out on the EVGP equipment which is self-manufactured. The experimental results of observation are agreement with simulation results. Thereby the kinematic model is proved to be valid. There are three stages which are fine lapping, super fine lapping and polishing in processing experiment. In the lapping stage, the variation of ball lot diameter, sphercity and the deviation of surface roughness $R a$ have been improved significantly and gradually to be convergence. After the final polishing stage, the best polishing results of a single ball achieved are surface roughness $R a$ of $9 \mathrm{~nm}$ and sphericity of $0.114 \mu \mathrm{m}$.
\end{abstract}

Key words: kinematics; processingtrajectory; consistency; eccentric variable-radius v-groove; precision balls

\section{0 前言}

球体作为高精度轴承、滚珠丝杜及直线导轨中 的关键元件常成组使用, 一批球的精度一致性将直

* 国家自然科学基金(U1401247, U1604254, 51375455)和浙江省公益公用 技术应用性(2017C31113)资助项目。20180309 收到初稿, 20180916 收 到修改稿
接影响这些零件的运动精度、噪音及寿命，进而影 响整个加工设备及装置的性能 ${ }^{[1-3]}$ 。超精密研磨抛光 是获得高精度球体的最终加工环节 ${ }^{[4]}$ 。目前, 球体 批量加工主要采用同心圆沟槽盘球体加工方法, 该 方法由日本学者INAGAKI 等 ${ }^{[5]}$ 于 1976 年首次提出, 其基本原理为球体置于两块磨盘之间，其中一块磨 盘上加工出同心圆 V 形槽, 另一块为平盘。球体在 磨盘旋转驱动下沿同心圆沟槽运动, 在研磨液的作 
用下实现球面材料去除。ZHANG 等 ${ }^{[6-7]}$ 基于运动学 原理对该方式下的球面加工轨迹进行了分析, 其理 论和实验结果均显示加工轨迹是固定圆环, 无法形 成球面。在实际加工中, 通过添加摚动或外循环装 置来随机改变球体被加工的滚道位置, 加工路径不 可控, 使得球面概率成形, 难以保证球体批量加工 的精度和尺寸一致性。

日本学者提出三转盘加工方法即将传统 $\mathrm{V}$ 形槽 加工方式中的沟槽盘于 $\mathrm{V}$ 形槽处分割开, 使整个机 构由三块可独立转动的磨盘组成, 加工过程中三块 盘同时旋转驱动球体自转并公转 ${ }^{[8-11]}$ 。袁巨龙等 ${ }^{[12-15]}$ 基于机构运动学原理分析三转盘加工方法后得出该 装置中的一个驱动是冗余的, 于是并结合研磨盘转 速变化的过程控制, 提出了双自转球体加工方法。 三转盘和双自转加工方式中加工轨迹均能够全包络 整个球面 ${ }^{[16-17]}$, 球体加工精度高, 但是加工装置装 球量少造成批加工效率较低。

针对上述问题, 袁巨龙等 ${ }^{[18]}$ 提出一种变曲率沟 槽球体加工方法, 以变曲率沟槽盘取代同心圆沟槽 盘, 使球体运动状态随沟槽曲率半径的变化而变化, 实现了与三转盘加工方式相同的加工轨迹均匀包络 球面效果。同时, 由于该加工方法采用了多沟道形 式, 在批加工能力上和同心圆沟槽盘球体加工方法 相当, 比只有单个沟槽的三转盘方式有了极大的提 高完全可以满足工厂的批量生产需求; 机械结构方 面, 三转盘加工设备内外盘的同轴度要求非常高, 且动力源多, 而该加工方法仅需一个动力源, 结构 简单, 易保证精度要求。采用该方法的明显优势是 每颗球体的加工路径一致能够保证高一致性, 但是 由于所采用的变曲率沟槽形状是螺旋槽, 曲率变化 小, 对球体自转角的影响范围小, 在此基础上本文 作者提出了偏心式变曲率沟槽加工方法。

本文首先阐述偏心式变曲率沟槽球体加工的基 本原理。随后, 基于纯滚动运动的假设条件, 建立 单颗球体的几何运动学模型, 并将几何运动学分析 结果结合旋转坐标变换, 仿真球面加工轨迹分布。 最后搭建实验平台, 对所建立的球体运动学模型进 行验证, 并对一批球体进行了加工试验研究。

\section{1 偏心式变曲率沟槽加工基本原理}

偏心式变曲率沟槽加工方法的基本原理如图 1 所示。该装置主要由平盘(下盘)、变曲率沟槽盘 (上盘)、加压装置、驱动装置组成。以阿基米德螺 旋线作为变曲率沟槽滚道的线型, 其极坐标表达 式为

$$
\rho=a \varphi(t)
$$

式中, $\rho$ 为极径; $\varphi(t)$ 为随时间变化的极角; $a$ 为沟 槽间距系数。变曲率沟槽的中心与磨盘中心存在偏 心距 $e$ 。

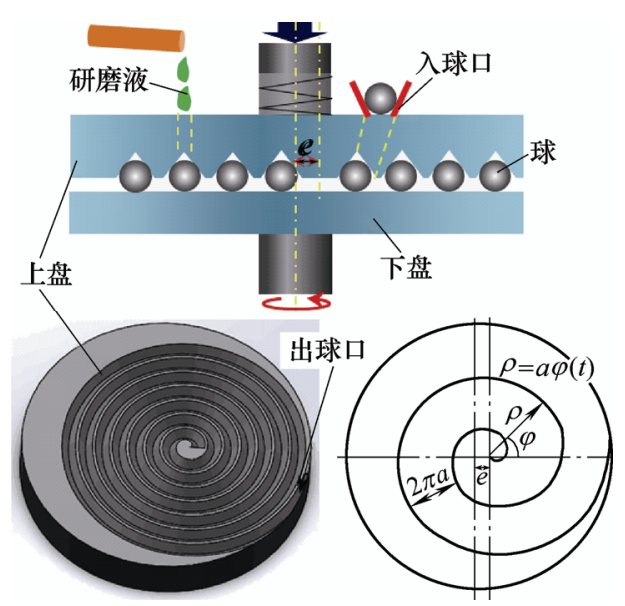

图 1 偏心式变曲率沟槽加工原理

加工过程中, 下盘旋转驱动球体依序沿变曲率沟 槽向外运动, 同时不断自转并绕旋转主轴公转, 上盘 实现对球体的精密加压, 在研磨液的作用下实现球面 材料的去除。球体从磨盘入球口经过整个变曲率沟槽 路径至磨盘出球口完成单个加工周期, 再由送料机构 从磨盘外沿的出球口依序送至沟槽中心的入球口实 现循环加工。因此, 单个加工周期的球体运动状态是 分析的关键, 此后均为该周期的不断叠加。

\section{2 球体几何运动学分析}

基于纯滚动运动理论分析, 首先作如下假设。

(1) 工件均为理想球体, 忽略加工过程中球与 球之间的推挤摩擦等相互作用。

(2) 球体与平盘接触点处为纯滚动, 与沟槽接 触点处为切向纯滚动。

(3) 球与磨盘均为刚体, 不考虑接触变形。

选取单颗球体进行几何运动学分析, 如图 2 所 示。球体与上下盘三接触点为 $A 、 B 、 C$, 以加工盘 主轴中心为坐标原点建立球-盘相对运动分析的全 局坐标系 $O_{1} X Y Z$, 坐标原点在主轴中心 $O_{1}$, 盘面上 为 $X$ 轴和 $Y$ 轴, $Z$ 轴垂直于盘面。 $O_{\mathrm{a}}$ 为任意时刻球 心所在沟槽滚道位置的瞬时曲率中心, 沿着该曲率 中心的方向, 接触点 $A 、 B 、 C$ 在一条直线上。沿着 该直线方向将球体切开, 从垂直于该直线的方向观 察如图 $2 \mathrm{~b}$ 所示。局部坐标系建立在球心与该时刻变 曲率中心连线的方向上, 以球心 $O_{\mathrm{b}}$ 为原点, 形成局 部坐标系 $O_{\mathrm{b}} x y z$ 如图 2c 所示, 为便于计算, 以球心 的两个速度分量为计算坐标系 $O_{\mathrm{b}} \tau n$ 。图 2 中各参数 
含义如表 1 所示。

表 1 几何运动学的符号及定义

\begin{tabular}{|c|c|c|c|}
\hline 符号 & 定义 & 符号 & 定义 \\
\hline$r_{\mathrm{b}}$ & 球体半径 & $\boldsymbol{V}_{\mathrm{ObO} 2}$ & 球心的速度矢量 \\
\hline$\omega_{1}, \omega_{2}$ & 下、上盘转速 & $\omega_{s}$ & 球体自转角速度 \\
\hline$l_{B}, l_{C}, l_{A}$ & $\begin{array}{l}\text { 磨盘上接触点至磨 } \\
\text { 盘中心的距离 }\end{array}$ & $\omega$ & $\begin{array}{l}\text { 球心相对于沟槽中心 } \\
\text { 的瞬时公转速度 }\end{array}$ \\
\hline$\alpha, \beta$ & 沟槽半角 & $\omega_{g}$ & $\omega_{s}$ 在 $Y$ 轴上的正交分量 \\
\hline$\varphi_{1}$ & $\begin{array}{l}\text { 面 } A O_{a} O_{b} \text { 与面 } A O_{b} O_{2} \\
\text { 间的夹角 }\end{array}$ & $\omega_{b}$ & $\begin{array}{l}\omega_{s} \text { 在球体经圆大剖面 } \\
\text { 内的正交分量 }\end{array}$ \\
\hline $\begin{array}{c}\boldsymbol{V}_{B O 2}, \boldsymbol{V}_{C O 2}, \\
\boldsymbol{V}_{A O 1}\end{array}$ & $\begin{array}{l}\text { 盘上接触点的速度 } \\
\text { 矢量 }\end{array}$ & $\theta$ & $\begin{array}{l}\text { 自转角速度分量 } \omega_{b} \text { 的 } \\
\text { 方位角 }\end{array}$ \\
\hline $\boldsymbol{V}_{O 2 O 1}$ & $\begin{array}{l}\text { 沟槽中心相对于磨 } \\
\text { 盘中心的速度矢量 }\end{array}$ & $\gamma$ & $\begin{array}{l}\text { 自转角速度分量 } \omega_{g} \text { 的 } \\
\text { 方位角 }\end{array}$ \\
\hline
\end{tabular}

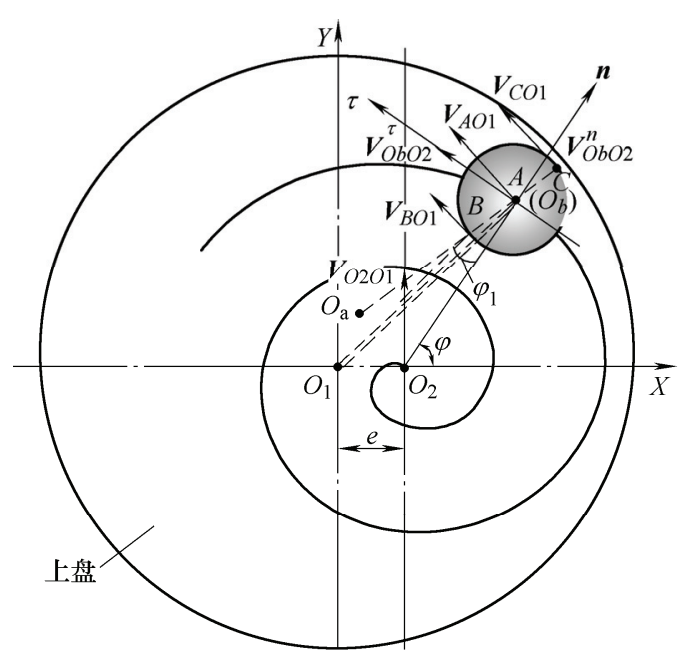

(a) 沿 $Z$ 方向的俯视图

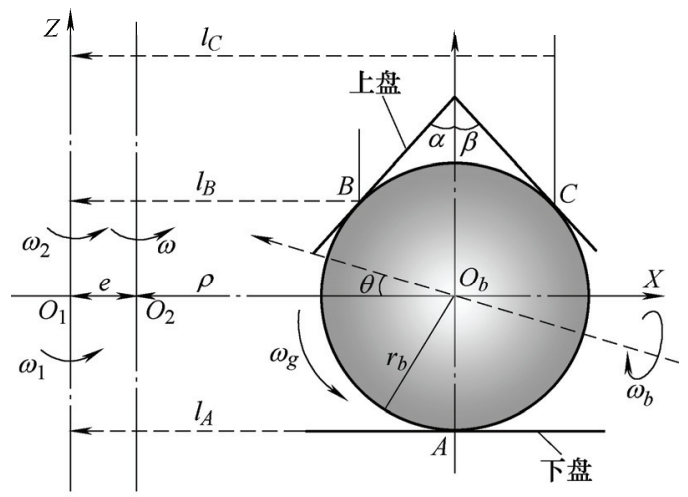

(b) 沿 $\tau$ 方向的正视图

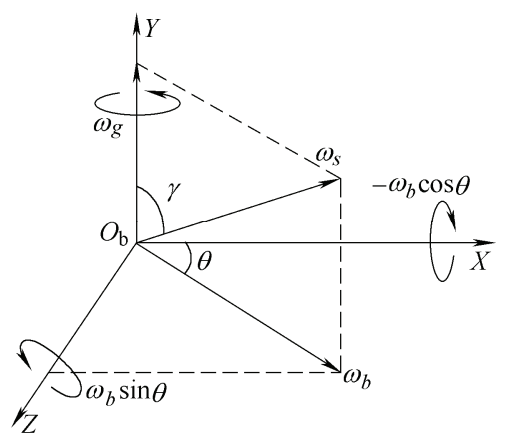

(c) 球心局部坐标系统

图 2 几何关系及坐标系统
基于纯滚动运动的假设条件, 速度矢量分析方 法用来分析三接触点在全局坐标系内的运动关系, 将图 2 中的速度矢量关系提取出来, 形成图 3 中各 个接触点的速度矢量图。则可得三接触点处的速度 矢量方程如式(1) (3)。

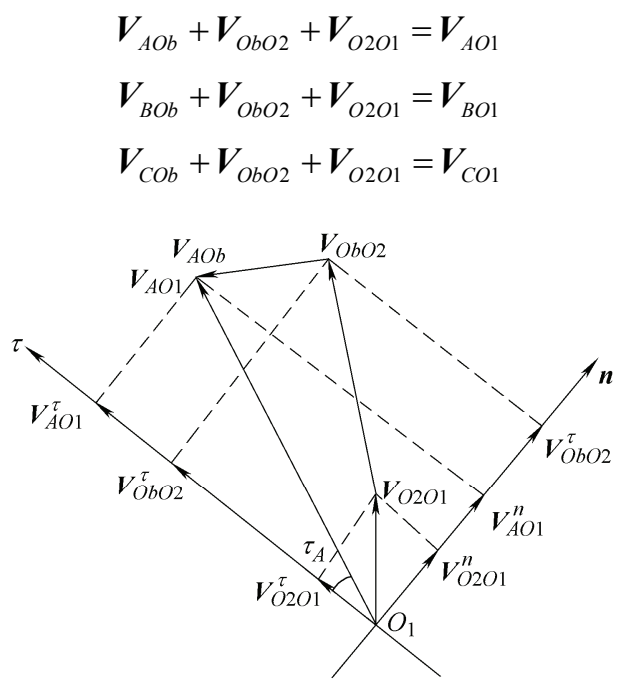

(a)

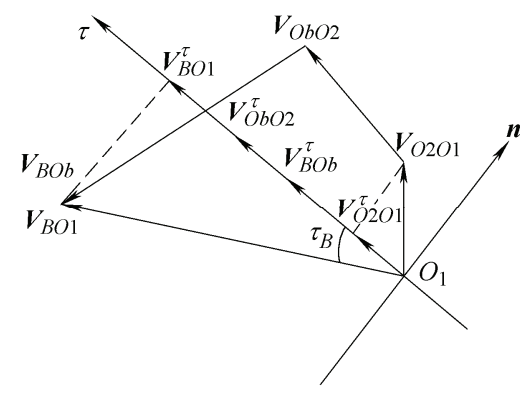

(b)

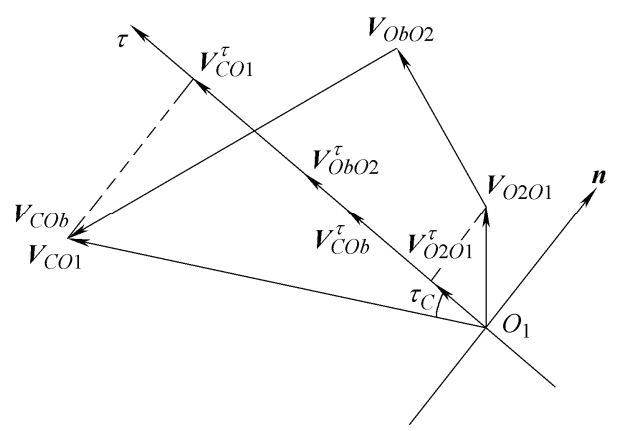

(c)

图 3 接触点 $A 、 B 、 C$ 速度矢量分析

如图 2a 所示, $A O_{a} O_{b}$ 面和 $A O_{b} O_{2}$ 面是分析接触 点速度矢量的两个关键面, $\varphi_{1}$ 为面 $A O_{a} O_{b}$ 与面 $A O_{b} O_{2}$ 间的夹角。变曲率线型中球心 $O_{b}$ 、瞬时曲率 中心 $O_{a}$ 及沟槽轨道形状起始点 $O_{2}$ 之间的关系可根 据相关数学知识求得。

球心 $O_{b}$ 在坐标系 $O_{1} X Y$ 中的投影方程如式(4)、 
(5)所列。

$$
\begin{gathered}
X_{O b}=\rho \cos \varphi \\
Y_{O b}=\rho \sin \varphi
\end{gathered}
$$

瞬时曲率中心 $O_{a}$ 的坐标 $(\varepsilon, \eta)$ 方程如式 $(6) 、(7)$ 所列。

$$
\begin{gathered}
\varepsilon=X_{O b}-\frac{Y_{O b}{ }^{\prime}\left(1+Y_{O b}{ }^{\prime 2}\right)}{Y_{O b}{ }^{\prime \prime}} \\
\eta=Y_{O b}+\frac{1+Y_{O b}^{\prime 2}}{Y_{O b}{ }^{\prime \prime}}
\end{gathered}
$$

此时, 变曲率线型中球心 $O_{b}$ 、瞬时曲率中心 $O_{a}$ 及变曲率沟槽中心 $O_{2}$ 三点间的长度关系可根据 余弦定理求得如式(8)。

$$
\cos \varphi_{1}=\frac{o_{a} o_{b}{ }^{2}+o_{2} o_{b}{ }^{2}-o_{2} o_{a}{ }^{2}}{2 \cdot o_{2} o_{b} \cdot o_{2} o_{b}}
$$

根据三角函数关系即可得式(9)。

$$
\sin \varphi_{1}=\frac{1}{\sqrt{1+\varphi^{2}}}
$$

$\boldsymbol{V}^{\tau}{ }_{\mathrm{ObO} 2}$ 和 $\boldsymbol{V}^{n}{ }_{\mathrm{ObO} 2}$ 为 $\boldsymbol{V}_{\mathrm{ObO} 2}$ 在 $\tau$ 方向和 $n$ 方向上的 两个分量, 则可得

$$
\begin{gathered}
\left|\boldsymbol{V}_{\mathrm{ObO} 2}^{\tau}\right|=\rho \omega \\
\left|\boldsymbol{V}_{\mathrm{ObO} 2}^{n}\right|=\tan \varphi_{1} \rho \omega=1 / \varphi \rho \omega
\end{gathered}
$$

$\boldsymbol{V}_{O 2 O 1}^{\tau}$ 为 $\boldsymbol{V}_{O 2 O 1}$ 在 $\tau$ 方向分量, 根据几何关系可得

$$
\left|\boldsymbol{V}_{O 2 O 1}^{\tau}\right|=e \omega_{2} \cos \varphi
$$

接触点 $A$ 在 $\tau 、 n$ 方向的分量

$$
\begin{gathered}
\left|\boldsymbol{V}_{A O 1}\right| \cos \tau_{A}=\left|\boldsymbol{V}_{A O 1}^{\tau}\right|=\omega_{1}(\rho+e \cos \varphi) \\
\left|\boldsymbol{V}_{A O 1}\right| \sin \tau_{A}=\left|\boldsymbol{V}_{A O 1}^{n}\right|=\omega_{1} e \cos \varphi
\end{gathered}
$$

$V_{B O 1}$ 和 $V_{C O 1}$ 在 $\tau$ 方向分量如下式

$$
\left|\boldsymbol{V}_{B O 1}^{\tau}\right|=\left|\boldsymbol{V}_{B O 1}\right| \cos \tau_{B}=\omega_{2} l_{B} \cos \tau_{B}
$$

$$
\begin{gathered}
\omega=\frac{\omega_{2} \rho(\cos \alpha+\cos \beta)-\omega_{2}\left(l_{3}+l_{1} \cos \varphi\right)(\cos \beta-\cos \alpha)+e\left(\omega_{1}-\omega_{2}\right) \cos \varphi \sin (\alpha+\beta)+\omega_{1} \rho \sin (\alpha+\beta)}{\rho(\cos \alpha+\cos \beta)+\rho \sin (\alpha+\beta)} \\
\omega_{g}=\frac{e\left(\omega_{1}-\omega_{2}\right)\left(\cos \varphi \sin \varphi_{1}-\sin \varphi \cos \varphi_{1}\right)+\omega_{1} \rho \sin \varphi_{1}}{r_{b}} \\
\theta=\arctan \left(\frac{\rho \omega_{2}(\sin \beta-\sin \alpha)-\rho \omega(\sin \beta-\sin \alpha)-\omega_{2}\left(l_{3}+l_{1} \cos \varphi\right)(\sin \alpha+\sin \beta)}{\rho\left(\omega_{2}-\omega\right)(\cos \alpha+\cos \beta)-\omega_{2}\left(l_{3}+l_{1} \cos \varphi\right)(\cos \beta-\cos \alpha)}\right) \\
\omega_{b}=\frac{\rho \omega_{2}(\sin \beta-\sin \alpha)-\rho \omega(\sin \beta-\sin \alpha)-\left(l_{3}+l_{1} \cos \varphi\right)(\sin \alpha+\sin \beta)}{r_{b} \sin \theta \cos \varphi_{1} \sin (\beta+\alpha)} \\
\gamma=\arctan \omega_{b} / \omega_{g}=\arctan \left(\frac{\rho \omega_{2}(\sin \beta-\sin \alpha)-\rho \omega(\sin \beta-\sin \alpha)-\omega_{2}\left(l_{3}+l_{1} \cos \varphi\right)(\sin \alpha+\sin \beta)}{\sin \theta \cos \varphi_{1} \sin (\beta+\alpha)\left(e\left(\omega_{1}-\omega_{2}\right)\left(\cos \varphi \sin \varphi_{1}-\sin \varphi \cos \varphi_{1}\right)+\omega_{1} \rho \sin \varphi_{1}\right)}\right)
\end{gathered}
$$


由式(25) (29)可知, 表征球体运动规律的自转 角速度和自转角 $\omega_{g} 、 \omega_{b} 、 \theta$ 和 $\gamma$ 与球径、V形槽半角、 磨盘转速、变曲率沟槽滚道极径和偏心距等因素均 有关。为避免不同球径影响使模型的适应性, 式子 右边的分子分母均除以球体半径 $r_{b}$, 使得表达式与 $\rho / r_{b}$ 和 $e / r_{b}$ 相关。

\section{3 加工轨迹}

\section{1 轨迹点仿真流程}

加工轨迹分布规律的分析是研究工件几何轮

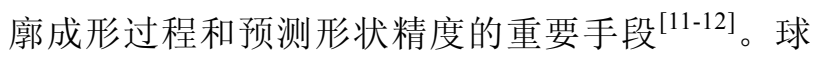
面加工轨迹包络整个加工球面且轨迹重复几率小 是精密球体成形的理论前提条件, 根据第 2 部分 球体运动学分析, 球面加工轨迹点计算流程如图 5 所示, 利用旋转坐标变换计算每时刻加工轨迹点 的坐标。

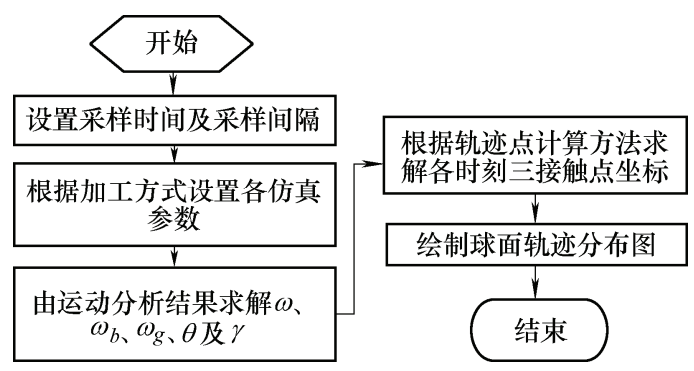

图 5 球面加工轨迹点计算流程图

\section{2 加工轨迹点的坐标计算}

以球心为原点建立坐标系, 参考图 2c 所示球体 自转系统的定义, 球体在变曲率沟槽运动的过程中, 球面与盘面接触点的位置不断变换, 首先假定三接 触点的初始坐标为

$$
\begin{gathered}
\boldsymbol{P}_{A}=\left[\begin{array}{lll}
0 & r_{b} & 0
\end{array}\right]^{\mathrm{T}} \quad \boldsymbol{P}_{B}=\left[\begin{array}{llll}
-r_{b} \cos \alpha & 0 & -r_{b} \sin \alpha
\end{array}\right]^{\mathrm{T}} \\
\boldsymbol{P}_{C}=\left[\begin{array}{llll}
r_{b} \cos \beta & 0 & -r_{b} \sin \beta
\end{array}\right]^{\mathrm{T}}
\end{gathered}
$$

将 $\boldsymbol{P}_{A}, \boldsymbol{P}_{B}$ 和 $\boldsymbol{P}_{C}$ 统一到一个矩阵 $\boldsymbol{P}_{0}$ 中, $\boldsymbol{P}_{0}=\left[\boldsymbol{P}_{A}\right.$, $\left.\boldsymbol{P}_{B}, \boldsymbol{P}_{C}\right]$ 。三接触点关于三个坐标轴的角速度分别为 $-\omega_{b} \cos \theta 、 \omega_{g} 、 \omega_{b} \sin \theta$, 则该时刻绕三坐标轴的旋转 角度如式(30) (32)。

$$
\begin{gathered}
\psi_{x}(i)=-\omega_{b}(i) \cos \theta(i) \Delta t \\
\psi_{y}(i)=\omega_{g}(i) \Delta t \\
\psi_{z}(i)=\omega_{b}(i) \sin \theta(i) \Delta t
\end{gathered}
$$

式中, $\psi_{x}(i) 、 \psi_{y}(i) 、 \psi_{z}(i)$ 表示该时刻的旋转角度; $i$ 为采样点; $\Delta t$ 为采样间隔。

假定初始旋转角度为零, 则下一时刻旋转角度 可表示为

$$
\begin{aligned}
& \Psi_{x}(i+1)=\Psi_{x}(i)+\psi_{x}(i) \\
& \Psi_{y}(i+1)=\Psi_{y}(i)+\psi_{y}(i) \\
& \Psi_{z}(i+1)=\Psi_{z}(i)+\psi_{z}(i)
\end{aligned}
$$

式中, $\Psi_{x}(i), \Psi_{y}(i)$ 和 $\Psi_{z}(i)$ 表示任意时刻绕 $X, Y, Z$ 轴的旋转角度。

根据坐标变换的原理, 得绕每个轴旋转矢量为

$$
\begin{aligned}
& \boldsymbol{X}_{r o t}=\left[\begin{array}{ccc}
1 & 0 & 0 \\
0 & \cos \Psi_{x}(i+1) & -\sin \Psi_{x}(i+1) \\
0 & \sin \Psi_{x}(i+1) & \cos \Psi_{x}(i+1)
\end{array}\right) \\
& \boldsymbol{Y}_{r o t}=\left[\begin{array}{ccc}
\cos \Psi_{y}(i+1) & 0 & \sin \Psi_{y}(i+1) \\
0 & 1 & 0 \\
-\sin \Psi_{y}(i+1) & 0 & \cos \Psi_{y}(i+1)
\end{array}\right] \\
& \boldsymbol{Z}_{r o t}=\left[\begin{array}{ccc}
\cos \Psi_{z}(i+1) & -\sin \Psi_{z}(i+1) & 0 \\
\sin \Psi_{z}(i+1) & \cos \Psi_{z}(i+1) & 0 \\
0 & 0 & 1
\end{array}\right]
\end{aligned}
$$

则可得每时刻球面加工轨迹点的旋转坐标值

$$
\begin{aligned}
& \boldsymbol{P}_{A}(i+1)=\boldsymbol{Y}_{r o t} \cdot \boldsymbol{X}_{r o t} \cdot \boldsymbol{Z}_{r o t} \cdot \boldsymbol{P}_{A}(i) \\
& \boldsymbol{P}_{B}(i+1)=\boldsymbol{Y}_{r o t} \cdot \boldsymbol{X}_{r o t} \cdot \boldsymbol{Z}_{r o t} \cdot \boldsymbol{P}_{B}(i) \\
& \boldsymbol{P}_{C}(i+1)=\boldsymbol{Y}_{r o t} \cdot \boldsymbol{X}_{r o t} \cdot \boldsymbol{Z}_{r o t} \cdot \boldsymbol{P}_{C}(i)
\end{aligned}
$$

根据实际经验设置仿真参数, 仿真条件如表 2 所示。

表 2 仿真条件

\begin{tabular}{lc}
\hline \multicolumn{1}{c}{ 参数 } & 参数 \\
\hline 轨迹方程 $/ \mathrm{mm}$ & $\rho=a \cdot \varphi ; a=1.5, \varphi \in(0,32 \pi)$ \\
球体半径 $r_{b} / \mathrm{mm}$ & 2.5 \\
偏心距 $e / r_{b}$ & 4 \\
槽型半角 $\alpha, \beta /\left({ }^{\circ}\right)$ & 45 \\
平盘转速 $\omega_{1} /(\mathrm{r} / \mathrm{min})$ & 15 \\
沟槽盘转速 $\omega_{2} /(\mathrm{r} / \mathrm{min})$ & 0.001 \\
采样间隔 $\Delta t / \mathrm{s}$ & 0.04 \\
\hline
\end{tabular}

单个加工周期后球面加工轨迹的分布如图 6 所 示, 三接触点处的球面加工轨迹能够包络整个球面, 且轨迹形态呈现多方向性。仿真结果从理论上说明 了偏心式变曲率沟槽球体加工的可行性。

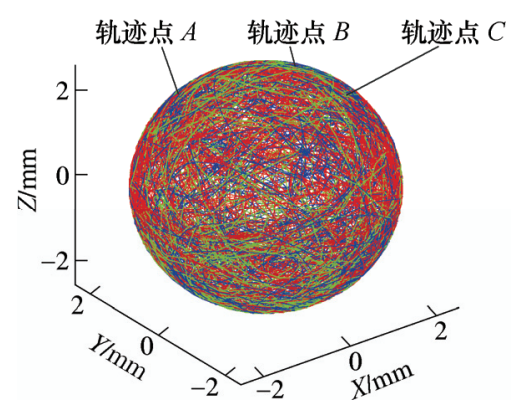

图 6 单个加工周期后球面加工轨迹的分布 


\section{4 加工试验}

偏心式变曲率沟槽球体加工试验装置如图 7 所 示, 该试验平台转速和压力可控。磨盘材料性能和 几何尺寸如表 3 所示。首先进行单周期球面加工轨 迹分布的验证试验, 将钢球置于硫酸铜溶液中, 使 球体表面产生易于与原来球体颜色区分的黄色铜。 为便于观察, 验证试验采用平盘表面贴上 1200 \#的 $\mathrm{SiC}$ 砂纸对表面附着铜的球体进行干磨, 如图 8 所 示, 磨盘转速为 $8 \mathrm{r} / \mathrm{min}$, 压力为 $5 \mathrm{~N} / \mathrm{Ball}$ 。

表 3 磨盘材料性能和几何尺寸

\begin{tabular}{lc||lc}
\hline \multicolumn{1}{c||}{ 参数 } & 数值 & \multicolumn{1}{c}{ 参数 } & 数值 \\
\hline 磨盘材料 & 球墨铸铁 & 盘径 $/ \mathrm{mm}$ & 300 \\
密度 $/\left(\mathrm{g} / \mathrm{cm}^{3}\right)$ & 7.3 & 沟槽角度 $/\left(^{\circ}\right)$ & 45 \\
耐磨性 & 良好 & 沟槽宽度 $/ \mathrm{mm}$ & 8.8 \\
硬度 $/ \mathrm{HB}$ & $130 \sim 220$ & 球体露出高度 $/ \mathrm{mm}$ & 6.138 \\
弹性模量 $/ \mathrm{GPa}$ & 154 & 沟槽间距 $/ \mathrm{mm}$ & 9.42 \\
泊松比 & 0.26 & 偏心距 $/ \mathrm{mm}$ & 15 \\
\hline
\end{tabular}

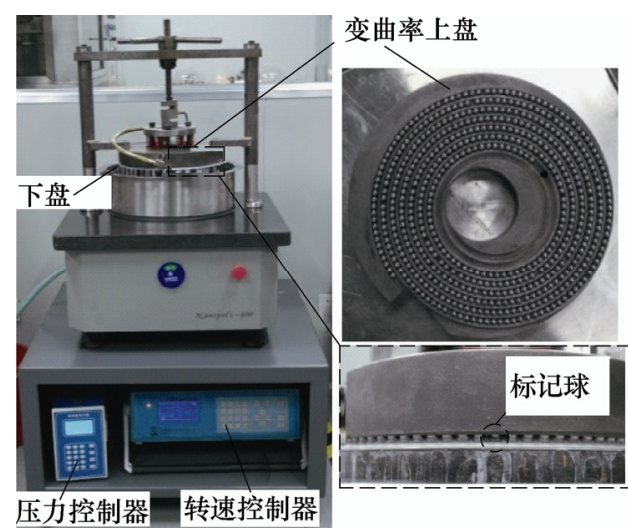

图 7 偏心式变曲率沟槽加工球体的试验场景

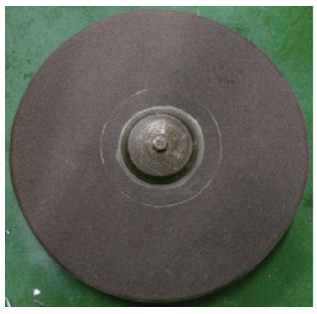

图 8 平盘表面贴砂纸

球体加工试验分精研 (8h)、超精研(12 h) 和抛光 (4 h), 加工条件如表 4 所示, 抛光液 ${ }^{[14]}$ 采用清华大 学科研人员研发的针对轴承钢的化学机械拋光液, 配比如表 5 所示。加工过程中, 直径 $8.5 \mathrm{~mm}$ 的 $\mathrm{Si}_{3} \mathrm{~N}_{4}$ 陶瓷球作为标记球, 不承受加工载荷, 每隔约 $2 \mathrm{~h}$, 选取该标记球后 10 粒球，对其直径变动量、圆度、 表面粗粘度 $R a$ 进行检测。球体质量检测采用 SI-234 精密天平，其精度为 $0.1 \mathrm{mg}$; 球体表面粗糙度 $R a$ 采用三丰(Mitutoyo)的 SJ-410 型表面粗糙度仪(采样
长度 $0.48 \mathrm{~mm}$ ), 每粒球体表面测量 3 个位置; 球度 误差采用三丰(Mitutoyo)的 RA-1500 型圆度仪, 对 每粒球体 3 个相互垂直方位的圆度进行检测, 取最 大值作为该球的球度误差。

表 4 加工试验条件

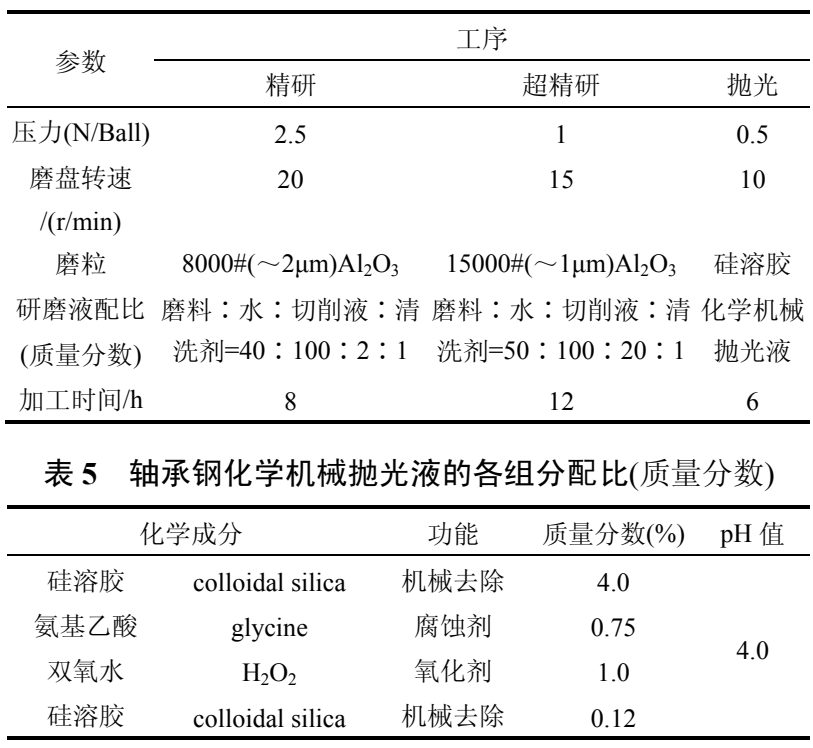

\section{5 加工结果与讨论}

\section{1 球面加工轨迹仿真模型验证}

如图 9 所示为偏心式变曲率沟槽单周期加工前 后球面的对比照片, 加工前钢球表面附着有均匀的 铜层, 经过单周期加工后球面的铜层全部去除, 表 明球-盘接触点遍历了整个球面, 进而宏观上验证了 偏心式变曲率沟槽球体加工中球面加工轨迹能够包 络整个球面的仿真结果, 说明了球体几何运动学分 析的有效性。

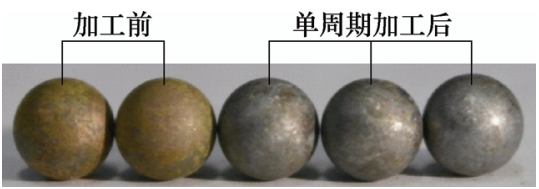

图 9 单周期加工前后球面对比

\section{2 加工质量及其影响因素分析}

利用精密天平测量出球体质量, 通过质量转换 成球体直径。图 10 为球体直径随加工时间的变化, 可以看出球体平均直径逐渐减小，精研、超精研的 平均材料去除率分别为 $1.25 \mu \mathrm{m} / \mathrm{h} 、 0.85 \mu \mathrm{m} / \mathrm{h}$, 抛光 阶段材料去除量很小，此阶段主要提升工件表面质 量。图 10 中偏差为该时刻所检测球体的批直径变动 量 $V_{D W L}$, 即最大球与最小球的平均直径之差。随着 加工进程的推进, $V_{D W L}$ 逐渐减小, 从初始 $1.08 \mu \mathrm{m}$ 降至最终的 $0.25 \mu \mathrm{m}$, 说明球体在直径上逐渐趋于一 
致, 其中超精研阶段 $V_{D W L}$ 变化最快。

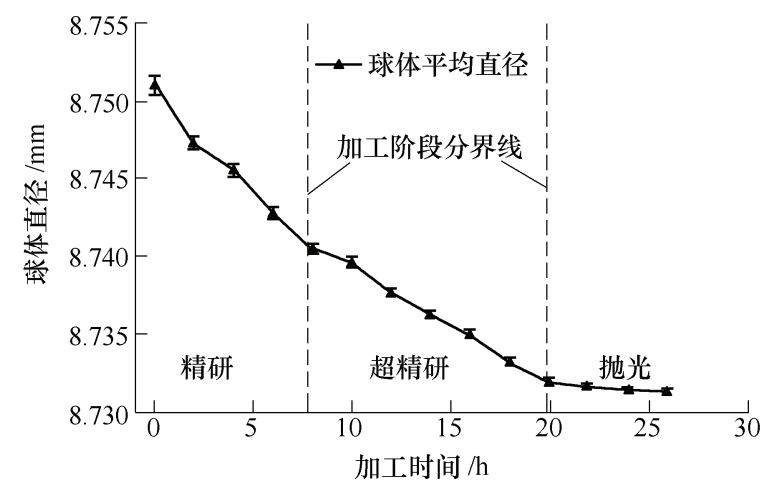

图 10 球体直径随加工时间的变化

图 11 所示为各加工阶段的球度误差平均值及 其偏差, 精研阶段的球度误差平均值收玫较快, 从 初始的 $0.237 \mu \mathrm{m}$ 降至 $0.184 \mu \mathrm{m}$, 经历超精研及抛光 后, 最终获得的球度误差平均值为 $0.122 \mu \mathrm{m}$ 。球度 误差偏差从初始的 $0.043 \mu \mathrm{m}$ 降至最终的 $0.014 \mu \mathrm{m}$ 。

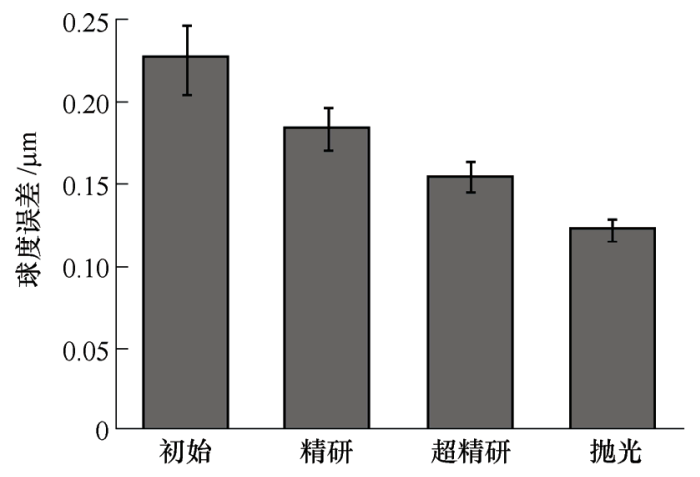

图 11 球度及其偏差随加工时间的变化

最终得到的最好球度值 $0.114 \mu \mathrm{m}$, 如图 12 所示。

圆度: $0.114 \mu \mathrm{m}$

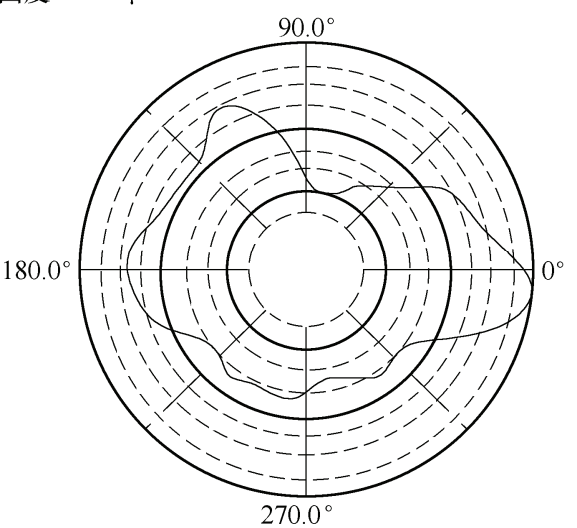

图 12 球度误差测量图

图 13 所示为各加工阶段的球体表面粗糙度 $R a$ 平均值及其偏差, 由于初始球体采用的是已拋光的 成品球, 经过精研后 $R a$ 平均值增大, 经过超精研 和抛光, $R a$ 从平均值 $26 \mathrm{~nm}$ 降至 $11 \mathrm{~nm}$ 。初始球体 $R a$ 偏差较大为 $15 \mathrm{~nm}$, 精研后尽管平均粗粘度值上 升, 但其偏差相较初始降至 $6 \mathrm{~nm}$, 之后的超精研和
抛光 $R a$ 平均值下降较明显, 其中抛光阶段表面粗 粘度的改善最明显，但粗粘度偏差变化不大，最终 的 $R a$ 偏差值为 $4 \mathrm{~nm}$ 。图 14 所示为单个球体最终球 面 $R a$ 达到的最小测量结果及其显微图片。图 15 所 示为最终加工完成的工件照片。

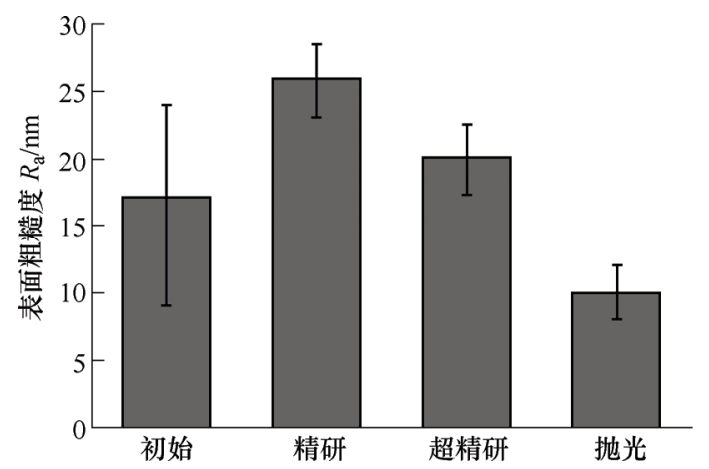

图 13 表面粗䊅度 $(R a)$ 及其偏差随加工时间的变化

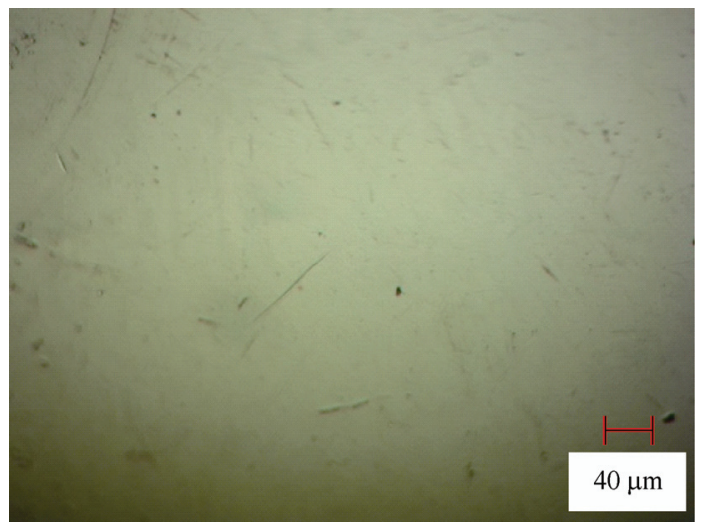

图 14 球面抛光后的显微图片(100x)

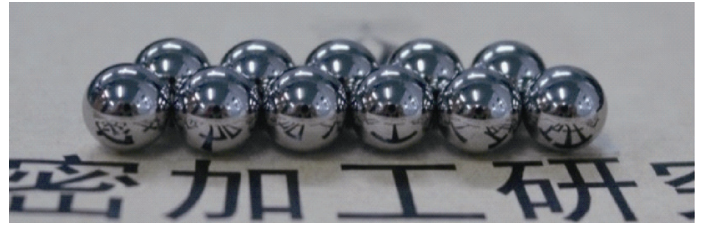

图 15 最终加工完成的工件照片

试验结果主要测试分析了球体平均直径及其偏 差、球度误差、表面粗粘度均值及其偏差的变化, 均值的逐渐改善证明该加工方法有利于改善球体精 度，偏差的收玫表明该加工方式有利于改善球体批 量加工的批一致性。因此，基于偏心式变曲率沟槽 球体加工方法能够获得高加工精度和批一致性的球 体。

\section{6 结论}

（1）基于纯滚动运动假设条件, 以几何运动学 为理论基础, 应用速度矢量法建立了单颗球体在偏 心式变曲率沟槽加工系统中的几何运动学模型, 推 导出球体各运动参数的表达式。 
(2) 根据球体运动状态, 结合坐标变换原理建 立了球面加工轨迹点的计算方法。利用仿真及试验 证实了该加工方法单周期加工后球面加工轨迹能够 包络整个球面。

(3) 进行了球体批量加工试验, 并对加工后球 体的直径、球度及表面粗糙度进行检测。结果得出: 球体的批直径变动量、球度偏差及表面粗粘度 $R a$ 偏差均得到改善并逐渐收玫, 得到最好的球度为 $0.114 \mu \mathrm{m}$, 表面粗粘度 $R a$ 为 $9 \mathrm{~nm}$ 。

\section{参 考 文 献}

[1] ZHUO Y, ZHOU X, YANG C. Dynamic analysis of double-row self-aligning ball bearings due to applied loads, internal clearance, surface waviness and number of balls[J]. Journal of Sound \& Vibration, 2014, 333(23): 6170-6189.

[2] BARZDAITIS V, BARZDAITIS V V, MASKVYTIS R, et al. New deep groove ball bearings high frequencies vibration testing[J]. Mechanika, 2014, 20(3): 287-293.

[3] NOGUCHI S, HIRUMA K, KAWA H, et al. The Influence of location of balls and ball diameter difference in rolling bearings on the nonrepetitiverunout (NRRO) of retainer revolution[J]. Precision Engineering, 2005, 29(1): 11-18.

[4] 袁巨龙, 张飞虎, 戴一帆, 等. 超精密加工领域科学技 术发展研究[J]. 机械工程学报, 2010, 46(15): 161-177. YUAN Julong, ZHANG Feihu, DAI Yifang, et al. Development research of science and technologies in ultra-precision machining field[J]. Journal of Mechanical Engineering, 2010, 46(15): 161-177.

[5] INAGAKI K, ABE K. Evaluation of performance of minute sphere grinders prepared for trial $[\mathrm{R}]$. Sendai : Tohoku University, 1976, 25, 1, 49-65.

[6] ZHANG B, NAKAJIMA A, NAKAJIMA A. Spherical surface generation mechanism in the grinding of balls for ultraprecision ball bearings[J]. ARCHIVE Proceedings of the Institution of Mechanical Engineers Part J Journal of Engineering Tribology, 2000, 214(4): 351-357.

[7] LEE R T, HWANG Y C, CHIOU Y C. Lapping of ultra-precision ball surfaces. Part I. Concentric V-groove lapping system[J]. International Journal of Machine Tools \& Manufacture, 2006, 46(10): 1146-1156.

[8] 黑部利次. ヤラミックスの球超精密研磨 [J]. 机械と 工具, 1990，34(2): 43-49.

KUROBE T. Super precision polishing of YARA mix ball. [J] Machine and Tool, 1990, 34(2): 43-49.

[9] KUROBE T, KAKUTA H, ONODA M. Spin angle control lapping of balls (1streport) - theoretical analysis of lapping mechanism[J]. Journal of the JapanSociety for
Precision Engineering, 1996, 62(12): 1773-1777.

[10] KUROBE T, KAKUTA H, ONODA M. Spin angle control lapping of balls (2streport) - theoretical analysis of lapping mechanism[J]. Journal of the Japan Society for Precision Engineering, 1997, 63(5): 726 730.

[11] KUROBE T, MORITA T, TSUCHIHASHI N. Super fine finishing of $\mathrm{Si} 3 \mathrm{~N}_{-} 4$ ceramic ball using spin angle controlled machining method[J]. Journal of the Japan Society for Precision Engineering Contributed Papers, 2004, 70(11): 1392-1396.

[12] 吕冰海. 陶瓷球双转盘研磨方式及成球机理的研究 $[D]$. 哈尔滨：哈尔滨工业大学, 2007

LÜ Binghai. Research on the rotated dual-plates lapping mode of ceramic ball and sphere-shaping mechanism[D]. Harbin: Harbin Institute of Technology, 2007.

[13] 冯铭. 螺旋式沟槽磨盘加工高精度球体的探索研究 [D]. 杭州: 浙江工业大学, 2013.

FENG Ming. A basis exploration on processing high-precision balls by spiral groovelapping plate[D]. Hangzhou: Zhejiang University of Technology, 2013.

[14] 郁炜, 吕冰海, 姚蔚峰, 等. 基于 ADMAS 的球体双自 转研磨方式下研磨盘转速优化研究 [J]. 中国机械工程, 2013, 24(7): 866-872.

YU Wei, LÜ Binghai, YAO Weifeng, et al. Speed optimization for lapping plates in RDP lapping mode based on ADAMS[J]. China Mechanical Engineering, 2013, 24(7): 866-872.

[15] YUAN J L, CHEN L N, ZHAO P, et al. Study on sphere shaping mechanism of ceramic ball for lapping process[J]. Key Engineering Materials, 2004, 259-260: 195-200.

[16] CHENG X, LIN F, SUN X, et al. Lapping motional trajectory analysis on sphere rotor of electrostatic gyroscope[J]. Manufacturing Technology \& Machine Tool, 2009, 30(9): 90-93.

[17] LIU D, DENG Q F, LV B H, et al. Simulation and analysis for spin angle track of the ball machined by dual rotation plates lapping method[J]. Applied Mechanics \& Materials, 2010, 37-38: 1148-1152.

[18] ZHAO P, GUO W, FENG M, et al. A novel lapping method for high precision balls based on variable-radius v-groove[J]. Journal of Micro and Nano-Manufacturing, 2013, 1(4): 041007.

作者简介: 郭伟刚, 男, 1980 年出生, 博士研究生, 教授。主要研究方 向为超精密加工技术及装备。

E-mail: guoweigang2004@126.com

袁巨龙, 男, 1962 年出生, 博士, 教授, 博士研究生导师。主要研究方 向为超精密加工技术及装备。

E-mail: jlyuan@zjut.edu.cn

赵萍(通信作者), 女, 1963 年出生, 副教授。主要研究方向为超精密加工 技术及装备。

E-mail: zhaoping@zjut.edu.cn 